\title{
Gene-gene Interaction of AhR with and within the Wnt Cascade Affects Susceptibility to Lung Cancer
}

\author{
Albert Rosenberger ( $\nabla$ arosenb@gwdg.de ) \\ Universitätsmedizin Göttingen https://orcid.org/0000-0001-7848-1332
}

Nils Muttray

Universitätsmedizin Göttingen: Universitatsmedizin Gottingen

Rayjean J Hung

Lunenfeld-Tanenbaum Research Institute

\section{David C Christiani}

Harvard University T H Chan School of Public Health

\section{Neil E Caporaso}

National Cancer Institute Division of Cancer Epidemiology and Genetics

Geoffrey Liu

Princess Margaret Hospital Cancer Centre

\section{Stig E Bojesen}

Herlev Hospital

\section{Loic Le Marchand}

University of Hawai'i at Mānoa John A Burns School of Medicine: University of Hawai'i at Manoa John

A Burns School of Medicine

\section{Demetrios Albanes}

National Cancer Institute

\section{Melinda C Aldrich}

Vanderbilt University Medical Center

\section{Adonina Tardon}

University of Oviedo Faculty of Medicine: Universidad de Oviedo Facultad de Medicina y Ciencias de la Salud

\section{Guillermo Fernández-Tardón}

University of Oviedo: Universidad de Oviedo

\section{Gad Rennert}

Carmel Medical Center

\section{John K Field}

Roy Castle International Centre for Lung Cancer Research: University of Liverpool North West Cancer Research Centre 
Roy Castle International Centre for Lung Cancer Research: University of Liverpool North West Cancer Research Centre

\section{Triantafillos Liloglou}

Roy Castle International Centre for Lung Cancer Research: University of Liverpool North West Cancer Research Centre

\section{Lambertus A Kiemeney}

Radboud Universiteit Nijmegen: Radboud Universiteit

\section{Philip Lazarus}

Washington State University - Spokane

\section{Bernadette Wendel}

Universitätsmedizin Göttingen

\section{Aage Haugen}

National Institute of Occupational Health

\section{Shanbeh Zienolddiny}

National Institute of Occupational Health

\section{Stephen Lam}

British Columbia Cancer Agency

\section{Matthew B Schabath}

H Lee Moffitt Cancer Center and Research Center Inc International Plaza

\section{Angeline S Andrew}

Dartmouth College Geisel School of Medicine

\section{Eric J Duell}

Catalan Institute of Oncology: Institut Catala d' Oncologia

\section{Susanne M Arnold}

Markey Cancer Center

\section{Gary E Goodman}

Swedish Medical Group

\section{Chu Chen}

Fred Hutchinson Cancer Research Center

\section{Jennifer A Doherty}

Huntsman Cancer Institute Cancer Hospital: University of Utah Health Huntsman Cancer Institute

\section{Fiona Taylor}

University of Sheffield English Language Teaching Centre: The University of Sheffield English Language Teaching Centre

\section{Angela Cox}

University of Sheffield Health Sciences Library: The University of Sheffield Faculty of Medicine Dentistry and Health

\section{Penella J Woll}

University of Sheffield 


\section{Angela Risch}

University of Salzburg amd Cancer Cluter Salzburg

\section{Thomas R Muley}

University Hospital Heidelberg: UniversitatsKlinikum Heidelberg

\section{Mikael Johansson}

Umea Universitet

\section{Paul Brennan}

IARC: International Agency for Research on Cancer

\section{Maria Teresa Landi}

National Cancer Institute

\section{Sanjay S Shete}

The University of Texas MD Anderson Cancer Center Pathology Department

\section{Christopher I Amos}

Baylor College of Medicine: Dan L Ducan Comprehensive Cancer Center

\section{Heike Bickeböller}

Universitätsklinikum Göttingen: Universitatsmedizin Gottingen

\section{INTEGRAL-ILCCO consortium}

Lunenfeld-Tanenbaum Research Institute

\section{Research}

Keywords: susceptibility, association, prediction, polygenic risk score, decision trees

Posted Date: September 23rd, 2021

DOI: https://doi.org/10.21203/rs.3.rs-658360/v2

License: @) (i) This work is licensed under a Creative Commons Attribution 4.0 International License. Read Full License 
Albert Rosenberger ${ }^{1}$, Nils Muttray ${ }^{1}$, Rayjean J Hung ${ }^{2,3}$, David C Christiani ${ }^{4}$, Neil E Caporaso ${ }^{5}$, Geoffrey Liu ${ }^{6,7}$, Stig E Bojesen , $^{8,9,10}$, Loic Le Marchand ${ }^{11}$, Demetrios Albanes ${ }^{5}$, Melinda C Aldrich ${ }^{12}$, Adonina Tardon ${ }^{13}$, Guillermo Fernández-Tardón ${ }^{13}$, Gad Rennert ${ }^{14}$, John K Field ${ }^{15}$, Michael P.A. Davies ${ }^{15}$, Triantafillos Liloglou ${ }^{15}$, Lambertus A Kiemeney ${ }^{16}$, Philip Lazarus ${ }^{17}$, Bernadette Wendel ${ }^{1}$, Aage Haugen ${ }^{18}$, Shanbeh Zienolddiny ${ }^{18}$, Stephen Lam ${ }^{19}$, Matthew B Schabath ${ }^{20}$, Angeline S Andrew ${ }^{21}$, Eric J Duell ${ }^{22}$, Susanne M Arnold ${ }^{23}$, Gary E Goodman ${ }^{24}$, Chu Chen ${ }^{25}$, Jennifer A Doherty ${ }^{26}$, Fiona TayIor $^{27}$, Angela Cox ${ }^{27}$, Penella J Woll ${ }^{27}$, Angela Risch ${ }^{28}$, Thomas R Muley ${ }^{29,30}$, Mikael Johansson ${ }^{31}$, Paul Brennan ${ }^{32}$, Maria Teresa Landi ${ }^{5}$, Sanjay S Shete ${ }^{33}$, Christopher I Amos ${ }^{34}$, Heike Bickeböller ${ }^{1}$ on behalf of the INTEGRAL-ILCCO consortium

1. Department of Genetic Epidemiology, University Medical Center, Georg-August-University Göttingen, Göttingen, Germany.

2. Lunenfeld-Tanenbaum Research Institute, Sinai Health System, University of Toronto, Toronto, Ontario, Canada.

3. Dalla Lana School of Public Health, University of Toronto, Toronto, Canada

4. Department of Environmental Health, Harvard T.H. Chan School of Public Health and Massachusetts General Hospital/Harvard Medical School, Boston, Massachusetts, USA.

5. Division of Cancer Epidemiology and Genetics, National Cancer Institute, US National Institutes of Health, Bethesda, Maryland, USA.

6. Medical Oncology and Medical Biophysics, Princess Margaret Cancer Centre, Toronto, Ontario, Canada.

7. Medicine and Epidemiology, Dalla Lana School of Public Health, University of Toronto, Toronto, Ontario, Canada.

8. Department of Clinical Biochemistry, Herlev and Gentofte Hospital, Copenhagen University Hospital, Copenhagen, Denmark.

9. Faculty of Health and Medical Sciences, University of Copenhagen, Copenhagen, Denmark.

10. Copenhagen General Population Study, Herlev and Gentofte Hospital, Copenhagen, Denmark.

11. Epidemiology Program, University of Hawaii Cancer Center, Honolulu, Hawaii, USA.

12. Department of Thoracic Surgery, Division of Epidemiology, Vanderbilt University Medical Center, Nashville, Tennessee, USA.

13. University of Oviedo, ISPA and CIBERESP, Faculty of Medicine, Oviedo, Spain.

14. Clalit National Cancer Control Center at Carmel Medical Center and Technion Faculty of Medicine, Haifa, Israel.

15. Roy Castle Lung Cancer Research Programme, The University of Liverpool, Department of Molecular and Clinical Cancer Medicine, Liverpool, UK.

16. Departments of Health Evidence and Urology, Radboud University Medical Center, Nijmegen, the Netherlands.

17. Department of Pharmaceutical Sciences, College of Pharmacy, Washington State University, Spokane, Washington, USA.

18. National Institute of Occupational Health, Oslo, Norway.

19. British Columbia Cancer Agency, Vancouver, British Columbia, Canada.

20. Department of Cancer Epidemiology, H. Lee Moffitt Cancer Center and Research Institute, Tampa, Florida, USA.

21. Department of Epidemiology, Geisel School of Medicine, Hanover, New Hampshire, USA.

22. Unit of Biomarkers and Susceptibility, Oncology Data Analytics Program, Catalan Institute of Oncology (ICO), Bellvitge Biomedical Research Institute (IDIBELL), Barcelona, Spain.

23. Markey Cancer Center, University of Kentucky, Lexington, Kentucky, USA.

24. Swedish Medical Group, Seattle, Washington, USA

25. Program in Epidemiology, Fred Hutchinson Cancer Research Center, Seattle, Washington, USA.

26. Huntsman Cancer Institute, Department of Population Health Sciences, University of Utah, Salt Lake City, Utha, USA

27. Department of Oncology and Metabolism, University of Sheffield, Sheffield, UK.

28. University of Salzburg and Cancer Cluster Salzburg, Salzburg, Austria.

29. Translational Lung Research Center (TLRC) Heidelberg, Member of the German Center for Lung Research (DZL), Heidelberg, Germany.

30. Translational Research Unit, Thoraxklinik, University Hospital Heidelberg,, Heidelberg, Germany.

31. Department of Radiation Sciences, Umeå University, Umeå, Sweden.

32. International Agency for Research on Cancer, World Health Organization, Lyon, France.

33. Department of Biostatistics, Division of Basic Sciences, The University of Texas MD Anderson Cancer Center, Houston, Texas, USA

34. Dan L Duncan Comprehensive Cancer Center, Baylor College of Medicine, Houston, Texas, USA.

Corresponding author: Albert Rosenberger, PhD

Universitätsmedizin Göttingen

Institut für Genetische Epidemiologie

Humboldtallee 32, 37073 Göttingen, Germany

Tel: +49 5513914044 ;

Fax: +49551 3914094;

Email: arosenb@gwdg.de

ORCID: 0000-0001-7848-1332

$54 \quad$ Running title: $\quad A h R / W n t$ genes in Lung Cancer

55 Key words: susceptibility, association, prediction, polygenic risk score, decision trees

56 Funding The National Institutes of Health (7U19CA203654-02/ 397 114564-5111078 Integrative Analysis of Lung 57 Cancer Etiology and Risk) supported this work. CARET is funded by the National Cancer Institute, National Institutes 
of Health through grants U01 CA063673, UM1 CA167462, R01 CA 111703, RO1 CA 151989, U01 CA167462 and funds from the Fred Hutchinson Cancer Research Center. The Boston Lung Cancer Study was funded by $\mathrm{NCl}$ grant 5U01CA209414. Other individual funding for participating studies and members of INTEGRAL-ILCCO are listed elsewhere. [1] The funders had no role in study design, data collection and analysis, decision to publish, or preparation of the manuscript. We acknowledge support by the Open Access Publication Funds of the Göttingen University.

Conflict of interest The authors declare no conflict of interest.

\section{Abstract}

Background: Aberrant Wnt signalling, regulating cell development and stemness, influences the development of many cancer types. The Aryl hydrocarbon receptor $(A h R)$ mediates tumorigenesis of environmental pollutants. Complex interaction patterns of genes assigned to $A h R / W n t$-signalling were recently associated with lung cancer susceptibility. Aim: To assess the association and predictive ability of $A h R / W n t$-genes with lung cancer in cases and controls of European descent. Methods: Odds ratios (OR) were estimated for genomic variants assigned to the Wnt agonist and the antagonistic genes $D K K 2, D K K 3, D K K 4, F R Z B, S F R P 4$ and Axin2. Logistic regression models with variable selection were trained, validated and tested to predict lung cancer, at which other previously identified SNPs that have been robustly associated with lung cancer risk could also enter the model. Further, decision trees were created to investigate variant $x$ variant interaction. All analyses were performed for overall lung cancer and for subgroups. $\underline{\text { Re- }}$ sults: No genome-wide significant association of $A h R / W n t$-genes with overall lung cancer was observed, but within the subgroups of ever smokers (e.g. maker rs2722278 SFRP4; OR=1.20; 95\%-Cl: 1.13-1.27; $p=5.610^{-10}$ ) and never smokers (e.g. maker rs1133683 Axin2; OR=1.27; 95\%-Cl: 1.19-1.35; $\mathrm{p}=1.010^{-12}$ ). Although predictability is poor, AhR/Wnt-variants are unexpectedly overrepresented in optimized prediction scores for overall lung cancer and for small cell lung cancer. Remarkably, the score for never-smokers contained solely two AhR/Wnt-variants. The optimal decision tree for never smokers consists of $7 \mathrm{AhR} / \mathrm{Wnt}$-variants and only two lung cancer variants Conclusions: The role of variants belonging to Wnt/AhR-pathways in lung cancer susceptibility may be underrated in main-effects association analysis. Complex interaction patterns in individuals of European descent have moderate predictive capacity for lung cancer or subgroups thereof, especially in never smokers. 
83

84

85

86

87

88

89

90

91

92

93

94

95

96

97

98

99

100

101

102

103

104

105

106

107

\section{Background}

Genome-wide association studies (GWAS) have identified dozens of susceptibility loci throughout the genome that are associated with lung cancer (LC) or one of its histological subtypes. [1-8] Genes related to Wnt signalling, one of the key pathway regulating cell development and stemness, were not detected as being associated to LC susceptibility in individuals of European descent so far, unlike TERT (5p15.33) that was one of the first for which a robust association was observed.[9] Aberrant Wht signalling is often observed in expression profiles of many cancers, but to date no association of Wnt/Ahr genes with susceptibility to cancer of any type has been observed.[10-12] Administration of RNAi against Wnt was shown to reduce tumour burden in lung adenocarcinoma (adenoLC). [13] In nonsmall cell lung cancer (NSCLC), overexpressed miR-582-3p maintains stemness features by negatively targeting the regulators of Wnt signalling Axin2, DKK3 and SRP1 for degradation, thereby increasing $\beta$-catenin mediated Wnt activity. [14] TERT expression was found to be directly enhanced by binding of $\beta$-catenin to its promoter region and thereby links telomerase activity to Wnt signalling. [10] This is inasmuch important, as TERT is one of the first and most robust susceptibility genes for LC identified by GWAS. $[15,16]$ The tight regulatory machinery of the Wnt pathway has several major antagonists, such as Secreted Frizzled related protein (sFRP), Dickopff 5 (DKK) protein and Axin2 protein. [17] Evidence also exists for a crosstalk between $A h R$ and Wnt signalling.[18]

Aryl hydrocarbon receptor $(7 \mathrm{p} 21.1 ; A h R)$ is a ligand induced transcription factor, which is translocated into the nucleus. It is known to mediate the toxicity and tumorigenesis of a variety of environmental pollutants, including for NSCLC. AhR upregulates the enzyme CYP1A1 when cells are exposed to carcinogenic metabolites, such as some polycyclic aromatic hydrocarbons (PAHs) found in cigarette smoke. The CYP1A1 coding gene is discussed as a suscepti-

bility gene for LC. AhR is a major determinant in the process of smoking driven LC. [19-21] The complexity of both the AhR signalling pathway and the Wnt signalling cascade is reflected by interaction effects of genomic variants within genes, which control their function. [22] Recently, the association of the Wnt-genes DKK4 (8p11.21), DKK3 (11p15.3), DKK2 (4q25), FRZB (2q32.1, also known as sFRP3), SFRP4 (7p14.1), Axin2 (17q24.1) and a potential interaction with $A h R$ was investigated with respect to the susceptibility to LC in a sample of 600 subjects from North India. [22, 23] A notable association with LC, e.g. for the SFRP4 variant rs1802073 (OR=3.19; 95\%-Cl 1.81-5.63), was 


\section{Selected Markers}

\section{Methods}

\section{Cases and Controls}

reported. Classification And Regression Tree (CART) analysis revealed an interaction of DKK2 and SFRP4 polymorphisms to be the best (off all investigated) predictors for LC; especially within smokers. They also reported to have identified several high-risk subgroups in smokers, e.g. characterised by DKK2 (rs17037102 / rs419558) and Axin2 (rs9915936). A similar picture was observed in a sample of 270 subjects from Istanbul, Turkey. [24] A two-way interaction between DKK3 (rs3206824) and SFRP4 (rs1802074) was found to be predictive of LC.

We aimed to assess a possible association of $A h R$ pathway and Wnt signalling cascade with LC within the large-scale series of cases and controls of European descent hold by the International Lung Cancer Consortium (ILCCO) / Integrative analysis of Lung Cancer Etiology and Risk (INTEGRAL). To do this, we also evaluated the contribution of these genes to genetic prediction of LC as a complement to known LC-related markers.

The work presented has been reviewed and approved by the ILCCO Steering Committee.

Phenotype and genotype data of 58,181 entries of the data repository of ILCCO were extracted. Details of the repository is described previously.[1, 25] QC control samples, individuals without information on smoking status or age, and samples of poor genotyping quality or sex discrepancies, were excluded, To avoid population stratification, this analysis is focused on European-ancestry population (defined as more than $95 \%$ probability of being of European descent). 14,068 incident LC-cases and 12,390 cancer-free controls of European descent remained for analysis. Those genotyped with other genome-wide array in addition to OncoArray were separated to form an independent validation set ( $2^{\text {nd }}$ validation set) of size ( $n=4,359$, including 2,360 LC-cases and 1,999 controls).

For this investigation we extracted the genotypes of 113 genomic variants (markers) assigned to 58 genes, previously robustly associated with the risk for LC or one of its histological subtypes [1-8] or proxies thereof (called LC-marker), and 296 markers assigned to 7 genes involved in Wnt signalling and listed in Bahl et al. [22, 23] and Yilmaz et al. [24] (called AhR/Wnt-marker). Thus, we focused this analysis to genes previously investigated with respect to LC. Fifty of these 409 markers were eliminated before analysis due to a $\mathrm{MAF}<1 \%$ (minor allele frequency), or departure from 
133

134

135

136

137

138

139

140

141

142

143

144

145

146

147

148

149

150

151

152

153

154

155

156

157

158

HWE (Hardy-Weinberg equilibrium) in genotypes (unaffected $p<10^{-7}$, affected $p<10^{-12}$ ), or low imputation accuracy (info<0.8). Seventy-eight of the remaining $L C$-markers were genotyped with the OncoArray (44 thereof are proxy SNPs identified using LDlink [26]) and 32 had to be imputed. Two-hundred twenty-one of the remaining AhR/Wntmarkers were genotyped and 28 have been imputed. A list of these markers extracted from ILCCO OncoArray repository is given in the appendix.

\section{Association analysis}

We first performed association analysis for each marker separately using the program PLINK. $[27,28]$ Crude (model 1) and adjusted odds ratios (ORs) were estimated along with 95\%-confidence intervals within log-additive models. Sex, age and smoking status and the first 3 principal components (PCs) to adjust for population stratification (model 2); and in addition the 6 most significantly associated LC-markers (rs55781567, 15q25.1 CHRNA5; rs11780471, 8p21.2 CHRNA2; rs7705526, 5p15.33 TERT; rs56113850, 19q13.2 CYP2A6; rs71658797, 1p31.1 AK5; rs11571833, 13q13.1 BRCA2) (model 3) were included in adjusted models. ORs were estimated for overall LC, small cell LC (SCLC), squamous cell LC (SqCLC), adenocarcinoma LC (adenoLC), ever smokers, never smokers and young individuals (21 to 55 years of age) as subgroups. We generated QQ-plots for the AhR/Wnt-markers and estimated the genomic inflation factor $\lambda$. To account for multiple testing, genome-wide statistical significance was considered to correspond to a $p$-value of $10^{-7}$ or lower, suggestive significance to a $p$-value between $10^{-5}$ and $10^{-7}$ and nominal significance to a p-value between 0.05 and $10^{-5}$.

\section{Logistic Regression - Predicting models with model selection}

We fitted logistic regression models with variable selection to find appropriate polygenic risk scores (PRS) in order to predict the disease (LC) status (affected or unaffected). Any AhR/Wnt-marker or the LC-marker could be included in the model without preference. To avoid multi-collinearity we removed one of two SNPs in LD to another $\left(R^{2}>0.8\right.$, pruning). The remaining entered the models as potential predictors. We performed forward selection until the Bayesian information criterion (BIC, most stringent selection), the Akaike information criterion (AIC, less stringent selection, contains in general more predictors) or the sample size corrected AIC (AICC) indicate a best solution (and 10 more selection steps). The resulting PRSs are called BIC-, AIC- and AICC-scores. Note, that for the purpose of model building, the AIC-selection is asymptotically equivalent to cross-validation (CV).[29, 30] To avoid overfitting, 
we assigned individuals to a training or a validation set (to build a score) and a testing set (to examine the score performance) with a $1 / 3$ probability each. For comparison, we also generated a $\mathrm{BIC}$-score with at least one marker, only allowing $L C$-markers to enter the model building. To compare the importance for LC prediction of the sets $g$ of LC-makers and AhR/Wnt-markers, respectively, we contrasted the importance-values defined as $I_{g}=\sum_{m \exists g}\left|\beta_{m}\right|$. $M A F_{m}$ for each score $\left(M A F_{m}\right.$ the minor allele frequency and $\beta_{m}$ the logistic regression coefficient of marker $m$ ). The superiority of the AIC-scores over the $\mathrm{BIC}^{\mathrm{LC}}$-score and the BIC-score was tested applying the nonparametric test of DeLong, DeLong, and Clarke-Pearson 1988 (1-sided) on AUCs of ROC (area under the receiver operation characteristic curve). [31] In addition, a corresponding precision-recall plot was created for the SCLC.

\section{Decision trees}

Decision trees were created to examine marker $\mathrm{x}$ marker interaction with respect to the LC prediction. Any AhR/Wntmarker or the LC-marker could be included in a tree without preference. This was accomplished in the entire sample and in all subgroups defined above. The R packages rpart and DescTools were used. [32, 33] To avoid trees being formed by spurious epistasis we removed one of two SNPs in $L D$ to another $\left(R^{2}>0.8\right.$, pruning). Since overfitting is a point of concern when building decision trees, the complexity parameter was first optimized applying 10-fold crossvalidation, grading the performance on the validation set by Somers' D (concordance of true and predicted LC-status). The ability of the optimal trees to predict the LC-status was then tested within the independent sample of 4,359 cases and controls. True positive (TP) and true negative (TN) rates are given.

All statistical analyses were performed with SAS ${ }^{\circledR}$ 9.4, PLINK 1.90 and 2.0 or R 4.0.2.

\section{Gene Expression}

We extracted information on gene expression from the Human Protein Atlas [34, 35] and LungGENS [36, 37].

\section{Results}

\section{Sample description}

The analysed sample consists of 14,068 LC-cases and 12,390 controls with median age of 63 . Sixty-three percent were male, $52 \%$ of cases and $28 \%$ of controls were current smokers. The most frequent histological subtype is adenocarcinoma (38\%), followed by squamous cell carcinoma (SqCLC) (26\%) and small cell lung cancer (SCLC) (10\%). The 
proportion of never-smokers was largest within the subgroup of adenocarcinoma cases (14\%), but almost the same between younger ( $<55$ years; $10 \%)$ and older (9\%) cases. Details on smoking status and histological subtypes are presented in Table 1.

Table 1 Smoking by LC status and subgroups

\section{Association analysis}

We first performed association analysis for each Wnt/AhR-marker separately. The p-values for an association of AhR/Wnt-markers with LC range from 0.005 (rs12115174; 8p11.21 DKK4; OR=0.9211) to 1 (model 2; adjusted for sex, age, smoking status and population stratification); with a negligible genomic inflation ( $\lambda=1.02)$. A nominally significant association $\left(10^{-5}<\mathrm{p} \leq 0.05\right)$ was observed for only 8 of the 249 markers $(\sim 3 \%)$. The corresponding point estimates of OR range from 0.88 (rs1053070054; 8p11.21 DKK4; $\mathrm{p}=0.007$ ) to 1.12 (rs74596148; 7p14.1 SFRP4; $\mathrm{p}=0.25)$. A QQ-plot indicates that achieved $\mathrm{p}$-values almost perfectly agree with the expectation of no associated marker (see Figure 1). P-values and OR are in moderate agreement between the models (e.g. model 2 to model 3; additionally adjusted by $L C$-markers: Kendall's rhop $=0.75$, rhooR $=0.78$ ).

\section{Figure 1:Association of AhR/Wnt-marker}

Subgroup analysis: When dividing the cases according to histological subtypes (SCLC; SqCLC and adenoLC) the observation of no detectable association for WNT/AhR-markers remains. Merely the number of nominally significant association $\left(10^{-5}<\mathrm{p} \leq 0.05\right)$ increases to $12(5 \%)$ or $21(8 \%)$ of the 249 markers for SqCLC and SCLC, respectively, hence close to the expected type 1 error. (Additional file 1: S-Table 2). When dividing the cases and controls according to their smoking behaviour (ever and never smokers), genome-wide significance $\left(p \leq 10^{-7}\right.$ ) was achieved for 7 and 8 markers, respectively. Another 12 and 3 markers, respectively, were found suggestively significant $\left(10^{-7}<p \leq 10^{-5}\right)$ (see Additional file 1: S-Figure 1) for ever and never smokers. Those markers found associated among ever smokers have mainly been directly genotyped and are assigned to SFRP4 and DKK4. E.g. for marker rs 2722278 we estimated an $\mathrm{OR}=1.20$ (95\%-Cl: $1.13-1.27)$, yielding a $\mathrm{p}$-value of $5.610^{-10}$. Those markers found associated among never smokers have mainly been imputed and are mostly assigned to $A x i n 2$, but also to $A H R, F R Z B$ and $D K K 2$. Marker rs17037102, assigned to DKK2, was the only one found associated with LC by Bahl et al. and in this analysis (see Table 2 and Additional file 1: S-Table 3). Interestingly, the ORs of these markers estimated by model 3 (additionally 
adjusted for selected LC-marker) differ from that estimated by model 2 . They are closer to one and no more significant. E.g. for rs1133683 (Axin2) we observe an OR=1.27 (95\%-Cl: 1.19-1.35, $\mathrm{p}=1 \times 10^{-12}$ ) fitting model 2, but OR=0.95 (95\%-Cl: 0.86-1.06, $\mathrm{p}=0.3586)$ fitting model 3 .

Table 2 Significantly associated AhR/Wnt-markers within never and ever smokers

\section{Logistic Regression - Predicting models with model selection}

We further fit logistic regression models with variable selection to evaluate the contribution of $A h R / W n t$-markers to a polygenic risk scores (PRS), but without postulating the usefulness of the score as such. Eight $L C$-markers from only eight LC-genes (CYP2A6, CHRNA5, TERT, AMICA1, CHRNA3, COPS2, HCG4 and CHRNA2) were selected for the BICscore (most stringent selection) to predict overall LC. Hence, the BIC-score and the BIC ${ }^{\mathrm{LC}}$-score are identical. In contrast, the AIC-score (for overall LC identical to the AICC-score) includes 20 LC-markers and remarkable $17 \mathrm{AhR} / \mathrm{Wnt}$ markers, with LC-markers being more important than the AhR/Wnt-markers (importance ratio 0.56: 0.34) (see Figure 2, Additional file 1: S-Figure 3 and S-Table 4). The ability to distinguish cases and controls from susceptibility genes only was, as expected, poor for each of the scores (see Additional file 1: S-Table 5). In the training set the performance of the AIC/AICC-score ( $A U C=0.607$ ) exceeded those of the $B I C / B I C{ }^{L C}$-score ( $A U C=0.582$ ) significantly ( $p<0.001$ ). Within the test set (AUCs: 0.577 and 0.576 ) and the $2^{\text {nd }}$ validation set (AUCs: 0.553 and 0.548 ), the higher complexity with additional $A h R /$ Wnt-markers did not improve discriminability for overall $L C(p=0.87$ and $p=0.35)$.

Similar score composition and performance was observed for most subgroups. The BIC-scores in the subgroups adenoLC (involved marker LC:AhR/Wnt=6:--), SCLC (3:--) and smokers (7:--) contained LC-markers only, whereas $A h R /$ Wnt-markers are included even under this stringent variable selection in the subgroups SqCLC $(5: 1)$ and Young (2:2). However, between 14 and 31 AhR/Wnt-markers entered these subgroup's AlC-scores. For these subgroups, the importance of the LC-markers for the AIC-score is higher than that of the included Ahr/Wnt-markers.

Figure 2: Comparison of score composition

Most important, we observed a significantly higher predictive accuracy (larger AUCs) of the AhR/Wnt-markers enriched AIC-scores compared to BIC ${ }^{L C}$-score in the subgroup of SCLC patients $\left(p=0.019 ; A U C_{A I C}=0.577 A U C_{B I C}=0.546\right)$ within the test set (see Additional file 1: S-Figure 4). For this subgroup, the selected AhR/Wnt-markers contribute to 
the AIC-score more than twice as much as the LC-markers (importance ration 0.60: 1.49). The precision-recall plot of Figure 3 indicates that a positive SCLC prediction based on the AIC-score can be trusted more than that based on LC-markers alone (BICC -score). In the $2^{\text {nd }}$ validation set the score-specific AUCs were similar but no more significantly different $\left(p=0.08\right.$; $A U C_{A I C}=0.564$ vs. $\left.A U C_{B I C}=0.531\right)$. The AIC-score of this SCLC-subgroup is composed of 12 LC-markers (assigned to CHRNA5, HCG4, DNAJB4 (4x each), CYP2A6, CHRNA3, CHRNA2, AMICA1, KCNJ4, AS1, BRCA2, EGFL8 and WNK1 (2x each)) and 27 AhR/Wnt-markers (assigned to all AhR/Wnt-genes except DKK3). However, only one LC patient in the test set $(n=434)$ and one in the $2^{\text {nd }}$ validation set $(n=164)$ was recognized as a patient at a threshold of $50 \%$ case probability.

Figure 3: ROC and precision-recall-plot: SCLC

Interestingly the BIC-score for never smokers was built by only two AhR/Wnt-markers (assigned to Axin2 and SFRP4) but not a single LC-marker. Further, the LC-markers are the minority in the composite of the AIC-score (15:23). They also contribute less to the AIC-score than the AhR/Wnt-markers (importance ratio of $0.96: 1.46$ ). The median predicted case probability, in the test set $(24.8 \%)$ and $2^{\text {nd }}$ validation set $(25.6 \%)$, exceeds that of controls by $1 \%$ - to $2 \%$ points. However, $A \cup C$ differed neither in the test set $\left(p=0.13 ; A \cup C_{A I C}=0.540 A \cup C_{B I C}=0.514\right)$ nor in the $2^{\text {nd }}$ validation set $\left(p=0.36 ; A \cup C_{A I C}=0.535 A \cup C_{B I C}=0.526\right)$ significantly. Nevertheless, this observation highlights the value of the AhR/Wnt-markers in the subgroup of never smokers.

\section{Decision trees}

Finally, we generated decision trees to evaluate the contribution of $A h R / W n t$-markers to LC prediction that allow for a complex interaction structure, but without postulating the usefulness of the trees as such. The decision tree for overall LC (whole sample) consists off solely a single decision node (rs55781567 assigned to CHRNA5), achieving a Somers' concordance index $\mathrm{D}=0.0565$ in the $2^{\text {nd }}$ validation set (see Additional file 1: S-Table 6 and S-Figure 2). A single-node decision-tree was also found optimal for young participants (split: rs1051730 assigned to CHRNA3), achieving a Somers' concordance index $\mathrm{D}=0.096$. These two, unsophisticated trees are characterised by balanced TP- (about 62\%) and TN-rates (about 44\%).

The decision trees for ever smokers, SCLC and SqCLC were more complex achieving Somers' concordance indexes D of $0.007,-0.0005$ and 0.0126 , respectively. The trees for SCLC and SqCLC are characterised by an extreme TP-rate 
$<5 \%$ and TN-rate $>99 \%$; the tree for Ever Smokers by a TP-rate $>99 \%$ and TN-rate $<5 \%$. Remarkably, a marker assigned to CHRNA5 was always chosen as the first and most important split for the trees for ever smokers, for SCC and SqCLC. However, markers assigned to AhR/Wnt-genes (smoker: DKK2; SCLC: FRZB; SqCLC; DKK2 and DKK3) appear at lower-level decision-nodes (Additional file 1: S-Figure 5, 6, 7 and 8). With the same program settings, no decision tree could be created for adenocarcinoma.

Most notable is the optimal decision tree for the 5,242 never smokers (75\% LC-cases, $25 \%$ controls), the only one that does not contain a marker belonging to the CHRN (Cholinergic receptors nicotinic subunits) gene group (see Figure 4). The tree is built from only two LC-markers but 7 AhR/Wnt-markers, achieving a Somers' concordance index $D=-0.002$. One can make out three branches of this tree. Branch I covers two thirds of individuals ( $n=754,66 \%$ of 1141 in the $2^{\text {nd }}$ validation set): All of these are graded as "unaffected" based on only the two LC-markers: first decision node (rs885518 assigned to MTAP) and second decision node (rs7705526 assigned to TERT that links telomerase activity to Wnt signalling). For branch II an additional node (rs17214897 assigned to DKK2) is taken into account, covering a further tenth $(9.9 \%)$ of never smokers. In this branch, very few subjects of the training set $(1.7 \%$ within branch II eq. $0.17 \%$ of all never smokers) are graded "affected". However, one in four individuals of the $2^{\text {nd }}$ validation set belonging to both branches, I and II, is truly "affected" but has not been detected (TP-rate=0\%, TN-rate=100\%). Rated as "affected" appears in the test set only in the third branch III, covering the remaining fourth of never smokers ( $n=284$ of the $2^{\text {nd }}$ validation set). This third branch requires genotypes of several $A h R / W n t-m a r k e r s$ assigned to $A H R$, Axin2, DKK2 and/or SFRP4. Herein, one in three ( $\mathrm{n}=97$ of the $2^{\text {nd }}$ validation set) is truly "affected" and is given a chance to be correctly identified, which appears in 8 LC-cases (TP-rate=9\%, TN-rate=88\%). We also noted that the histological subtypes are equally distributed between the branches (see Additional file 1: S-Table 7).

Figure 4:Decision tree for never smoker

\section{Gene Expression}

$A H R, A x i n 2, D K K 3$ are ubiquitously expressed, with RNA expression detected in many tissues and evidence for protein expression. Axin2 and $D K K 3$ are moderately to highly expressed in normal lung tissues according to the Human Protein Atlas. [34] AhR is expressed at low levels in macrophage cells of the lung. No expression is reported for other 
Wnt/AhR-genes. (see Additional file 1: S-Figure 9 and S-Table 9). Significant differential expression is listed in LungGENS for AhR, Axin2 DKK2, DKK3 and SFRP4 [36] (see Additional file 1: S-Table 8). Further, AhR is reported to be abundantly expressed in solid lung tumours, especially in adenocarcinomas. AhR overexpression was associated with upregulation of IL-6 secretion, which is critical for lung cancer initiation. [38] Detailed information on gene expression is given in the Appendix. In addition, the DKK1 serum level was seen as significantly lower in NSCLC and SCLC patients compared to healthy controls. [39] Significant upregulation of DKK2 expression was found in APC (adenomatous polyposis coli)-mutated non-SCLC lung cancers. [40]

\section{Discussion}

This investigation was intended to discover association of the Wnt-genes DKK4 (8p11.21), DKK3 (11p15.3), DKK2 (4q25), FRZB (2q32.1, also known as sFRP3), SFRP4 (7p14.1), Axin2 (17q24.1) and a potential interaction with AhRgenes, to LC in a large sample of 26,458 individuals of European descent. No marginal association of $A h R /$ Wnt-markers with overall LC was observed. Interestingly, an accumulation of associated markers was observed splitting the sample by smoking status, where respective markers in ever smokers are assigned to SFRP4. On the other hand, association analysis in never smokers reflects complex gene-gene interactions, as markers of several Ahr/Wnt-genes were found to be genome-wide associated with LC. This complexity is also visible through the decision tree analysis.

Recently, marginal associations of the AhR/Wnt-markers were reported for lung cancer case from North India, although in a much smaller sample of about 600 individuals. $[22,23]$ A notable association with LC, e.g. for the SFRP4 variant rs1802073 (OR=3.19; 95\%-Cl 1.81-5.63), was observed. Classification and Regression Tree (CART) analysis revealed an interaction of DKK2 and SFRP4 polymorphisms to be the best (off all investigated) predictors for LC; especially within smokers. They also reported several high-risk subgroups in smokers, e.g. characterised by DKK2 (rs17037102 / rs419558) and Axin2 (rs9915936). A similar picture has also been observed in a sample of 270 subjects from Istanbul, Turkey. [24]

We failed to directly replicate the single marker associations reported by Bahl et al. [22, 23] (North India) and Yilmaz et al. [24] (Turkey). The Indian population is known to be a mixture of several subpopulations [41], which can result in spurious associations. E.g. for rs7396187 assigned to DKK3 Bahl et al. reported a protective effect (OR GC+CC vs 
$311 \mathrm{cc}=0.63,95 \%-\mathrm{Cl}: 0.44-0.91, \mathrm{p}=0.01)$; however, along with a significant departure from HWE in controls $\left(\chi^{2}=15.11\right.$,

$312 \mathrm{df}=2, \mathrm{p}=0.001)$. In contrast, our analysed sample was carefully examined for ethnic homogeneity and principal com-

313 ponents were used to adjust for population stratification. The reports by Bahl et al. and Yilmaz et al. are themselves

314 contradictory in some details.

Yilmaz at al. reported a two-way interaction between DKK3 (rs3206824) and SFRP4 (rs1802074) to be predictive of LC. Among other constellations, Bahl et al. reported that DKK3 and SFRP4 were placed closely to each other by a Multifactor dimensionality reduction (MDR) for overall LC, while two markers of SFRP4 were closely placed within smokers. In contrast, markers assigned to $A x i n 2$, but also to $A H R, F R Z B$ and $D K K 2$ were observed as associated within never smokers. According to Bahl et al. markers of Axin2 and DKK2 were in never smokers closely placed by a MDR, too. The discrepancy between the total sample and the subsample association estimates point to smoking mediated associations.

Our analysis agrees with both previous studies in that complex interaction patterns between the investigated genes contribute to LC susceptibility as entirety or within specific subgroups. To discover patterns of Ahr/Wnt-genes involved in LC genesis we further changed the focus from significance of association to inclusion in prediction models, and followed two approaches: First, we searched for polygenic risk scores (PRS). Doing so, we add up marker main effects to construct multidimensional scores, optimising model fit (instead of marker preselection by $p$-value below some threshold), in order to discriminate cases from controls in a somehow ideal way. Complex gene $x$ gene (GxG) interactions are not modelled.

Nevertheless, the proportion of Ahr/Wnt-genes entering some of the predictive models was remarkable large, given that these markers are not, all other candidates however genome-wide significantly associated to LC. This was particularly noticeable for SCLC, since $A h R / W n t$-markers contribute more than twice as much to the prediction score as LC-markers. It is known, that within current smokers, tobacco consumption is strongest associated to SCLC. [42] Moreover, within never smokers, a stringed defined score is made up from only two AhR/Wnt-markers, assigned to Axin2 and SFRP4. However, the discriminative ability of PRSs for LC, contributing markers with significance for main effect at different levels, is in general poor. The AUC of the $\mathrm{BIC}$ LC score for overall LC $(0.58$ in the test set and 0.55 in the $2^{\text {nd }}$ validation set) corresponds to the $A U C=0.54$ based on four top $L C$-genes in a simulated population, as given 
by the GWAS-ROCS Database (https://gwasrocs.ca/). This may be due to other overpowering risk factors, since mod-

338 els including e.g. age, sex and smoking variables achieve higher AUCs (0.62 to 0.79). [43]

Recently two polygenic risk scores (PRSs) for overall-LC had been developed, validated and assessed with respect to improving eligibility to low-dose computed tomography (LDCT) as the only recommended screening test for lung cancer. Jia et al. [44, 45] build a PRS on 19 genome-wide associated SNPs $\left(p<0.510^{-8}\right)$. Hung et al. [46], integrated their PRS on 128 SNPs (35 "known" LC-related loci, 93 suggestive associated loci selected by LASSO-regression model) into the $\mathrm{PLCO}_{\text {all2014 }}$ risk model. Both approaches have been validated using data from the UK Biobank. For both scores, the mean PRS differed only slightly between LC cases and cancer-free controls (Jia: effect size 0.19; Hung: effect size $\sim 0.22$ ). For both scores, no substantial increase in discriminability of cases from controls is reported, when adding the PRS to existing risk models (Jia: family history - AUC=0.589, family history + PRS AUC=0.615; Hung: $\mathrm{PLCO}_{\text {all2014 }}-\mathrm{AUC}=0.828, \mathrm{PLCO}_{\text {all2014 }}+\mathrm{PRS}-\mathrm{AUC}=0.832$ ). However, both were able to show that the age at which a smoker crosses the recommended screening threshold of $1.5 \%$ for the 5 -year LC risk depends on the genetic background, which is sufficiently quantified by the PRS examined. Some smokers will be eligible by $<50$ years of age, others by> 60 years of age. Hence, constructing reliable PRS, even with small discriminability, may help to improve the performance of LDCT.

Two- and multiway GxG interaction can also contribute to LC susceptibility, rather than just markers with observed (marginal) main effects. GxG interaction is in general less commonly investigated, not only because this requires much larger samples. However, Li et al. [47] found RGL1:RAD51B in overall LC and non-SCLC, SYNE1:RNF43 in adenocarcinoma and FHIT:TSPAN8 in SqCLC to interactively contribute to LC susceptibility. As in the presented data analysis, the impact of these genes would also have been overlooked considering main effects only. Another reason could be that LC itself is just a generic term of several subcategories that differ in terms of LC initiation and require separate PRSs. $[43,48]$ A third reason of the poor performance may be due to the exclusively concentration on genetic effects, rather than modelling lifelong interaction with the environment as well. E.g. GxE interaction effects for LC have been observed smoking [49], exposure to asbestos fibres [50, 51] and exposure to radon [52, 53]. 
361 With this in mind, the data analysis presented shows that the complex interaction of Wnt-related genes has the 362 potential to be part of an adequate risk assessment for never-smokers or in relation to certain histological subtypes 363 of LC.

364
As a second approach, we constructed decision trees, which mainly depict GxG interaction patterns. Although, the ability to discriminate cases from controls is again poor, CHRNA5 was in general the most important first node for overall LC and in many subgroups. Ahr/Wnt-genes play a complex but important role in at least one quarter of never smokers, as seen before. Remarkably, TERT, which links telomerase activity to Wnt signalling, was central in that branch and important for the remaining three quarters of never smoker. This corresponds to a concentration of relevant genes for this subgroup in the CLPTM1L-TERT region on chromosome 5, as previously reported by Hung et al.. [54] Our observations confirm the suspicion, that LC in never smokers is a different entity, justified beforehand on differences in epidemiological, clinical and molecular characteristics. [48]

We would like to emphasize that this study was not intended to provide a definitive and reliable risk assessment, but rather aimed to examine in depth the LC-relevant complex interaction pattern of AhR/Wnt-genes hypnotized by Bahl et al.. Indeed, considering prediction instead of association provides weaker evidence for this, but is valid in view of the large amount of external evidence. The importance of the Wnt-signalling pathway and its antagonist's $S F R P, D K K s$ and $A x i n 2$ for cancer is outlined in the introduction. One can also assume a connection with the molecular functionality, since involved genes are expressed ubiquitously or in lung tissues. In summary, we were unable to replicate previously reported associations of Wnt/AhR-markers with LC. However, we observed a small but significant impact of these genomic variants on PRSs or decision trees to predict LC.

\section{Conclusions}

The role of markers belonging to Wnt signalling and the AhR pathway in LC susceptibility may be underrated in maineffects association analysis. Complex interaction patterns in individuals of European decent have moderate predictive capacity for LC or subsets thereof, especially in never smokers.

\section{List of abbreviations}

AhR

Aryl hydrocarbon receptor 


\begin{tabular}{|c|c|c|}
\hline 386 & GWAS & genome-wide association studies \\
\hline 387 & LC & lung cancer \\
\hline 388 & NSCLC & non-small cell lung cancer \\
\hline 389 & SCLC & small cell lung cancer \\
\hline 390 & $\mathrm{SqCLC}$ & squamous cell lung cancer \\
\hline 391 & adenoLC & adenocarcinoma lung cancer \\
\hline 392 & OR & odds ratio \\
\hline 393 & CART & classification and regression tree \\
\hline 394 & AUCs of ROC & area under the receiver operation characteristic curve \\
\hline 395 & ILCCO & International Lung Cancer Consortium \\
\hline 396 & INTEGRAL & Integrative analysis of Lung Cancer Etiology and Risk \\
\hline 397 & PRS & polygenic risk scores \\
\hline 398 & $\mathrm{BIC}$ & Bayesian information criterion \\
\hline 399 & $\mathrm{AIC}$ & Akaike information criterion \\
\hline 400 & $\mathrm{CV}$ & cross validation \\
\hline 401 & MAF & minor allele frequency \\
\hline 402 & $\mathrm{TP}$ & true positive rate \\
\hline 403 & $\mathrm{TN}$ & true negative rate \\
\hline 404 & LDCT & low-dose computed tomography \\
\hline
\end{tabular}


405 Declarations

\section{Ethics approval and consent to participate}

407 All participants in this study signed an informed consent, approved by the local internal review board or ethics com-

408 mittee and administered by trained personnel. All consortium research received approval from the Dartmouth Com409 mittee for Protection of Human Subjects on 7/30/2014 with id STUDY00023602. All experimental protocols and

410 other methods used comply with institutional, national, or international guidelines.

411 Consent for publication

$412 \quad$ Not applicable

\section{Availability of data and materials}

414 The data that support the findings of this study are available from ILCCO/INTEGRAL but restrictions apply to the 415 availability of these data, which were used under license for the current study, and so are not publicly available. Data are however available from the authors upon reasonable request and with permission of ILCCO/INTEGRAL.

\section{$417 \quad$ Competing interests}

418 The authors declare that they have no competing interests

\section{Funding}

420 The National Institutes of Health (7U19CA203654-02/ 397 114564-5111078 Integrative Analysis of Lung Cancer Eti-

421 ology and Risk) supported this work. CARET is funded by the National Cancer Institute, National Institutes of Health 422 through grants U01 CA063673, UM1 CA167462, R01 CA 111703, RO1 CA 151989, U01 CA167462 and funds from the 423 Fred Hutchinson Cancer Research Center. Other individual funding for participating studies and members of INTE424 GRAL-ILCCO are listed elsewhere $[10,30]$. The funders had no role in study design, data collection and analysis, 425 decision to publish, or preparation of the manuscript.

\section{Authors' contributions}

427 A. R. designed the investigation, carried out parts of the formal analysis and wrote the main manuscript text. N.M. 428 carried out parts of the formal analysis, prepared figures and critical reviewed and revised the manuscript. B.W. 
430 data curation and funding acquisition. H.B: supervised the investigation, including funding acquisition. R.J.H., H.B.,

431 L.LM., C.I.A. and L.A.K. critical reviewed and revised the manuscript. A.C., A.H., A.R., A.S.A., A.T., C.C., D.A., D.C.C.,

432 E.J.D., F.T., G.E.G., G.F.-T., G.L., G.R., H.B., J.A.D., J.K.F., L.A.K., L.LM., M.B.S., M.C.A., M.J., M.P.A.D., M.T.L., N.E.C.,

433 P.B., P.J.W., P.L., R.J.H., S.E.B., S.L., S.M.A., S.S.S., S.Z., T.L. and T.R.M. collected and provided study materials and

434 data.

435 Acknowledgements

$436 \quad$ Not applicable

437 Supplementary Information

438 "Additional file 1.pdf" contains additional information on a) which marker were extracted from ILCCO OncoArray

439 repository, b) single marker association, c) Polygenic Risk Scores (PRS) and Decision Trees and d) gene expression in

440 normal tissue.

441 Tables and figures

442 Table 1 Smoking by LC status and subgroups

\begin{tabular}{|c|c|c|c|c|c|c|c|c|c|c|}
\hline & & \multicolumn{3}{|c|}{ Never smoker } & \multicolumn{6}{|c|}{ Ever smoker } \\
\hline & & \multirow{2}{*}{$\begin{array}{l}\text { Total } \\
\quad \mathrm{N}\end{array}$} & \multicolumn{2}{|c|}{ never } & \multicolumn{2}{|c|}{ former } & \multicolumn{2}{|c|}{ current } & \multicolumn{2}{|c|}{ ever\$ } \\
\hline & & & $\mathrm{n}$ & $\%$ & $\mathrm{n}$ & $\%$ & $\mathrm{n}$ & $\%$ & $\mathrm{n}$ & $\%$ \\
\hline \multirow[t]{4}{*}{ control } & teenager & 6 & 3 & $50 \%$ & -- & -- & 3 & $50 \%$ & -- & - \\
\hline & young & 2,756 & 948 & $34 \%$ & 698 & $25 \%$ & 893 & $32 \%$ & 217 & $8 \%$ \\
\hline & old & 9,628 & 2,960 & $31 \%$ & 3,572 & $37 \%$ & 2,568 & $27 \%$ & 528 & $5 \%$ \\
\hline & all & 12,390 & 3,911 & $32 \%$ & 4,270 & $34 \%$ & 3,464 & $28 \%$ & 745 & $6 \%$ \\
\hline \multirow[t]{7}{*}{ case } & $\mathrm{SqCLC}$ & 3,692 & 138 & $4 \%$ & 1,257 & $34 \%$ & 2,158 & $58 \%$ & 139 & $4 \%$ \\
\hline & $\mathrm{SCLC}$ & 1,450 & 48 & $3 \%$ & 383 & $26 \%$ & 965 & $67 \%$ & 54 & $4 \%$ \\
\hline & other LC & 3,629 & 405 & $11 \%$ & 1,200 & $33 \%$ & 1,820 & $50 \%$ & 204 & $6 \%$ \\
\hline & AdenoLC & 5,297 & 740 & $14 \%$ & 1,989 & $38 \%$ & 2,401 & $45 \%$ & 167 & $3 \%$ \\
\hline & young & 2,765 & 281 & $10 \%$ & 452 & $16 \%$ & 1,945 & $70 \%$ & 87 & $3 \%$ \\
\hline & old & 11,303 & 1,050 & $9 \%$ & 4,377 & $39 \%$ & 5,399 & $48 \%$ & 477 & $4 \%$ \\
\hline & all & 14,068 & 1,331 & $9 \%$ & 4,829 & $34 \%$ & 7,344 & $52 \%$ & 564 & $4 \%$ \\
\hline total & & 26,458 & 5,242 & $20 \%$ & 9,099 & $34 \%$ & 10,808 & $41 \%$ & 1,309 & $5 \%$ \\
\hline
\end{tabular}



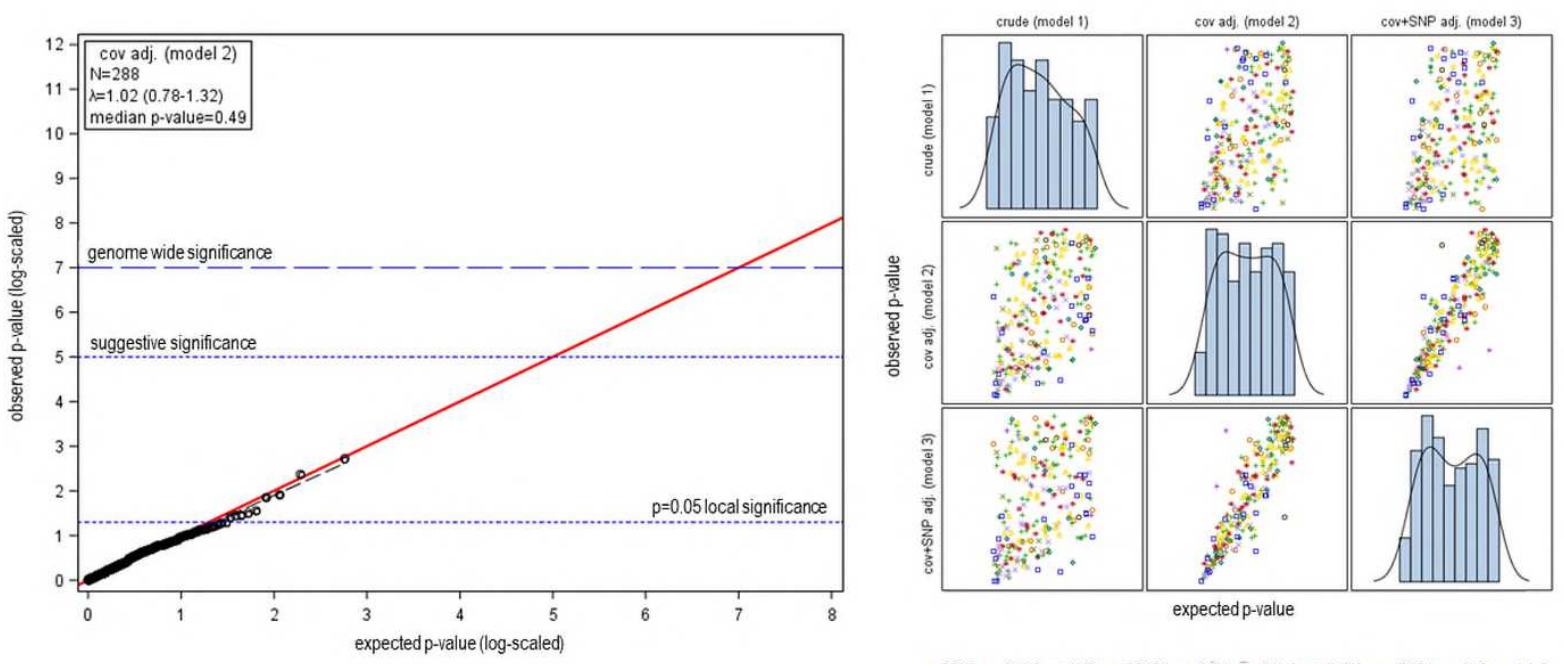

Left panel: QQ-Plot for model 2 (adjusted for sex, age and smoking status and the first three principal components); right panel: matrix of p-

values generated by model 1 (crude), model 2 (adjusted for sex, age and smoking status and the first three principal components) and model 3

significance: $10^{-5}<p$-value $\leq 0.05$.

Table 2 Significantly associated AhR/Wnt-markers within never and ever smokers

\begin{tabular}{|c|c|c|c|c|c|c|c|c|c|}
\hline \multirow[t]{2}{*}{ SNP } & & \multirow[t]{2}{*}{ Cyto band } & \multirow[t]{2}{*}{ MAF } & \multirow[t]{2}{*}{ gene } & \multicolumn{3}{|c|}{ model 2} & \multirow{2}{*}{$\begin{array}{c}\text { model } 1 \\
\text { OR }\end{array}$} & \multirow{2}{*}{$\begin{array}{c}\text { model } 3 \\
\text { OR }\end{array}$} \\
\hline & & & & & $p$-value & OR & $95 \%-\mathrm{Cl}$ & & \\
\hline \multicolumn{10}{|c|}{ never smoker } \\
\hline imputed & rs202198518\$ & $7 p 21.1$ & $14 \%$ & $A H R$ & $3.410^{-13}$ & 0.72 & $0.66-0.79$ & 0.71 & $0.90^{\text {n.s. }}$ \\
\hline imputed & rs2237297\$ & & $14 \%$ & & $9.910^{-14}$ & 0.71 & $0.65-0.78$ & 0.71 & $0.90^{\text {n.s. }}$ \\
\hline imputed & rs1133683 & $17 q 24.1$ & $42 \%$ & Axin2 & $1.010^{-12}$ & 1.27 & $1.19-1.35$ & 1.27 & $0.95^{\text {n.s. }}$ \\
\hline imputed & rs2240307 & & $5 \%$ & & $7.710^{-24}$ & 0.41 & $0.34-0.49$ & 0.40 & $0.62^{\text {n.s. }}$ \\
\hline imputed & rs35285779§ & & $9 \%$ & & $3.210^{-22}$ & 0.58 & $0.52-0.65$ & 0.58 & $1.10^{\text {n.s. }}$ \\
\hline imputed & rs35415678§ & & $9 \%$ & & $3.710^{-19}$ & 0.62 & $0.56-0.69$ & 0.62 & $1.10^{\text {n.s. }}$ \\
\hline imputed & rs288326 & $2 q 32.1$ & $10 \%$ & $F R Z B$ & $2.510^{-8}$ & 1.42 & $1.25-1.60$ & 1.41 & $0.98^{\text {n.s. }}$ \\
\hline imputed & rs17037102 & $4 q 25$ & $15 \%$ & $D K K 2$ & $7.410^{-15}$ & 0.69 & $0.63-0.76$ & 0.69 & 1.09 n.s. \\
\hline \multicolumn{10}{|c|}{ ever smoker } \\
\hline genotyped & rs12532321 & $7 p 14.1$ & $45 \%$ & SFRP4 & $1.310^{-9}$ & 1.14 & $1.09-1.19$ & 1.15 & $1.13^{\text {s.s. }}$ \\
\hline genotyped & rs7811872 & & $36 \%$ & & $1.310^{-8}$ & 0.88 & $0.84-0.92$ & 0.88 & $0.88^{\text {gw.s. }}$ \\
\hline genotyped & rs10226308 & & $42 \%$ & & $1.810^{-8}$ & 0.88 & $0.85-0.92$ & 0.89 & 0.89 gw.s. \\
\hline genotyped & rs10488617 & & $42 \%$ & & $1.610^{-8}$ & 0.88 & $0.85-0.92$ & 0.89 & 0.89 gw.s. \\
\hline genotyped & rs2722278 & & $16 \%$ & & $5.610^{-10}$ & 1.20 & $1.13-1.27$ & 1.16 & 1.20 gw.s. \\
\hline genotyped & rs2722279 & & $11 \%$ & & $9.010^{-9}$ & 1.22 & $1.14-1.31$ & 1.17 & 1.23 gw.s. \\
\hline genotyped & rs7811420 & & $43 \%$ & & $7.910^{-8}$ & 0.89 & $0.85-0.93$ & 0.89 & 0.89 gw.s. \\
\hline imputed & rs2073664 & 8p11.21 & $9 \%$ & DKK4 & $9.410^{-11}$ & 1.20 & $1.14-1.27$ & 1.15 & $1.08^{\text {s.s. }}$ \\
\hline
\end{tabular}

452 MAF: minor allele frequency; model 1: crude odds ratio (OR); model 2: adjusted for sex, age and smoking status and the first three principal

453 components; model 3: OR additional adjusted for 6 selected LC-markers. gw.s. genome-wide significant ( $p$-value $\leq 10^{-7}$ ); s.s. suggestive significant

$454\left(10^{-7}<p\right.$-value $\left.\leq 10^{-5}\right) ;{ }^{\text {n.s. }}$ not significant $(p>0.05)$. Only markers are listed for which genome-wide significance $\left(p-v a l u e ~ \leq 10^{-7}\right)$ was achieved.

$455 \$, \$$ pair of markers in $L D\left(R^{2}>0.8\right.$ in Populations of European decent) 


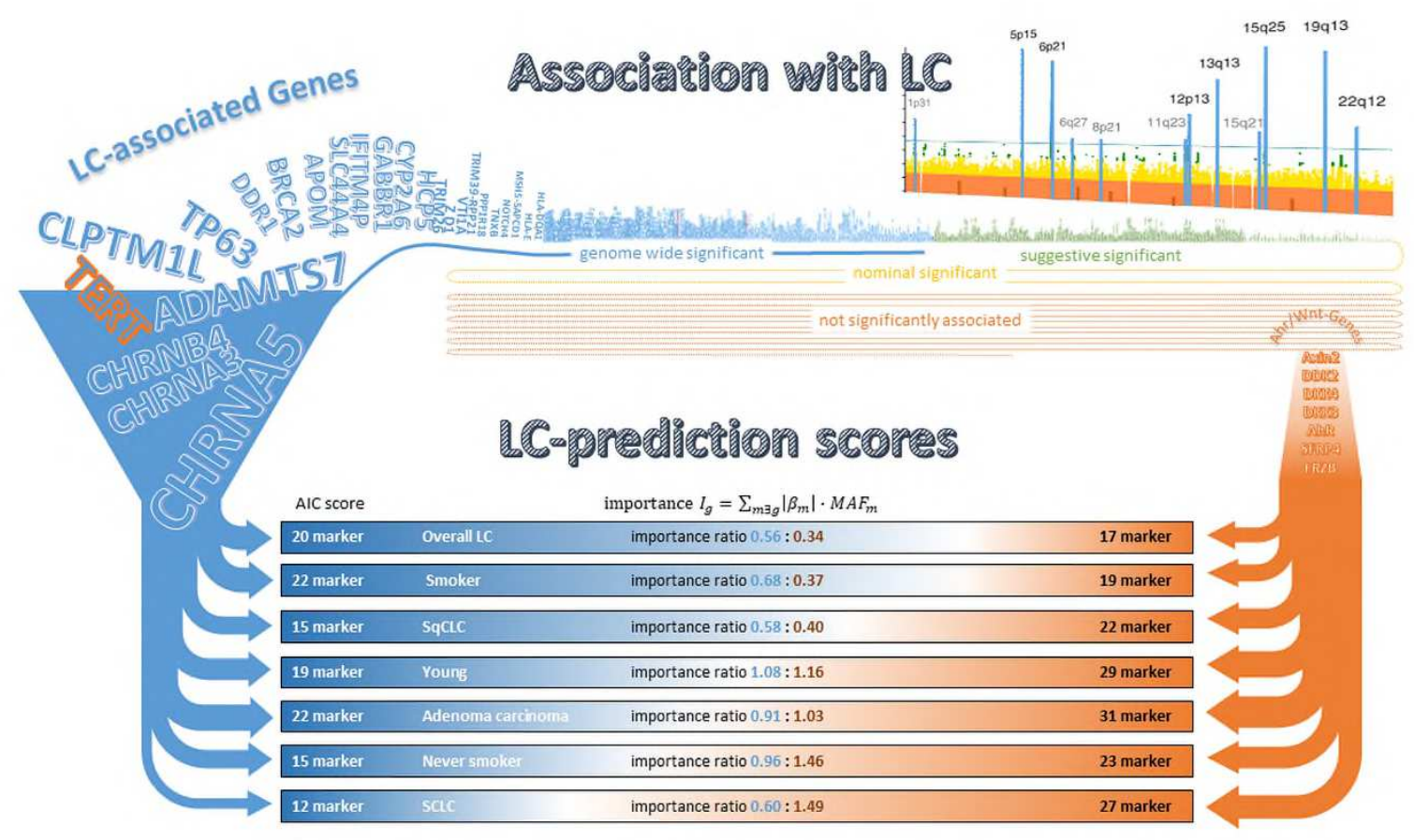

457

LC: lung cancer; AIC score: score of a logistic regression model with variant selection according to the Akaike information criterion (AIC); MAFm: 
Small cell lung cancer (SCLC)
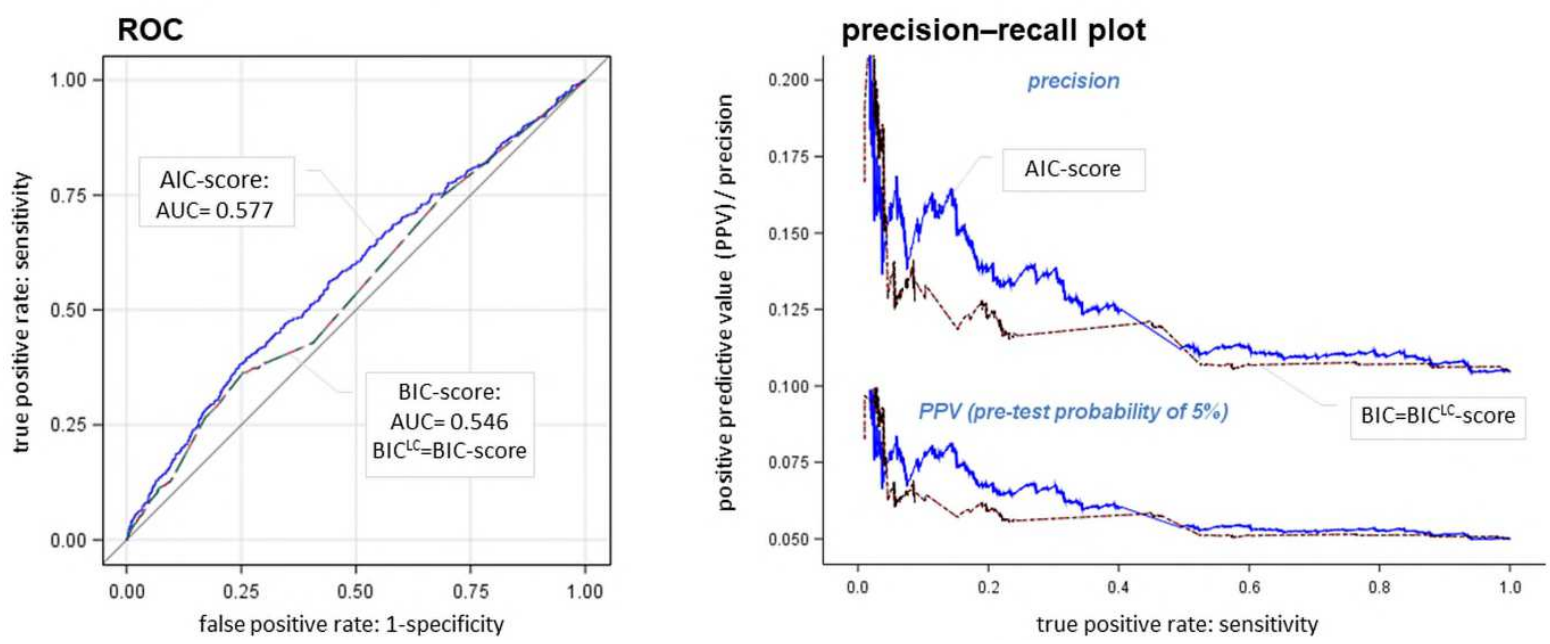

The diagnostic performance of the AIC-score compared to the BIC/BIC ${ }^{\mathrm{LC}}$-score in the test-set is presented. Left panel: ROC (receiver operation characteristics); right panel: corresponding precision-recall plot; precision =(true positive cases) / (true positive cases + false positive controls), positive predictive value $(\mathrm{PPV})=($ sensitivity $\times$ pre-test-probability) $/$ [(sensitivity $\times$ pre-test-probability $)+(1$-specificity $\times 1$-pre-test-probability)] 


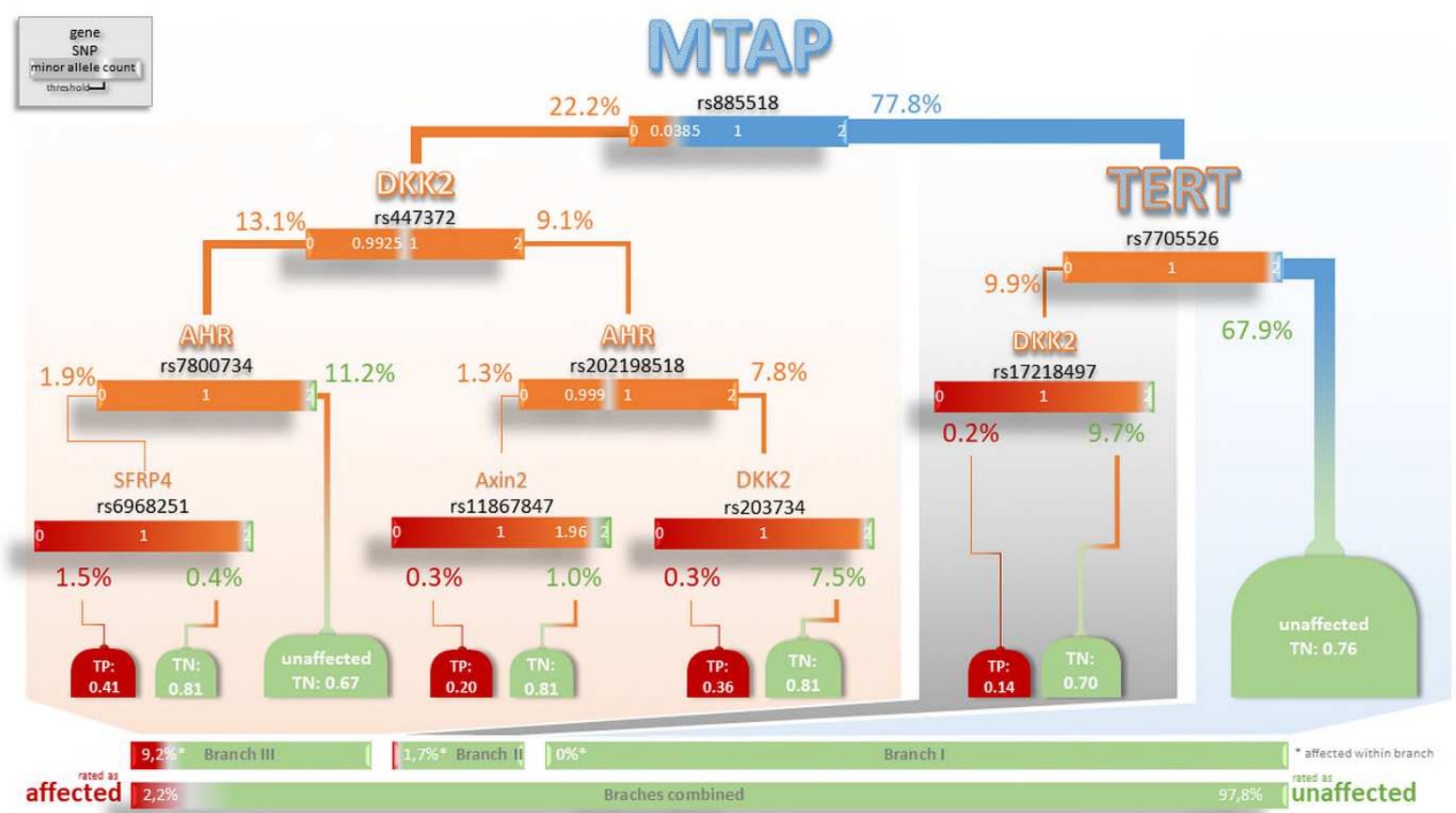

Node information: gene name, marker; split information below the node: threshold for minor allele count; blue split nodes: LC-genes, orange split nodes: Ahr/Wnt-genes, ; TERT is framed in orange because telomerase activity is related to Wnt signalling; decision nodes and bars: green for unaffected; red for affected, TN true negative rate, TP true positive red; the size of gene names, lines and decision notes is proportional to the size of the respective (sub)sample 


\section{References}

1. McKay JD, Hung RJ, Han Y, et al (2017) Large-scale association analysis identifies new lung cancer susceptibility loci and heterogeneity in genetic susceptibility across histological subtypes. Nat Genet 49:1126-1132

2. Timofeeva MN, Hung RJ, Rafnar T, et al (2012) Influence of common genetic variation on lung cancer risk: meta-analysis of 14900 cases and 29485 controls. Human molecular genetics 21:4980-95

3. Rosenberger A, Sohns M, Friedrichs S, et al (2017) Gene-set meta-analysis of lung cancer identifies pathway related to systemic lupus erythematosus. PLOS ONE 12:e0173339

4. Brenner DR, Brennan P, Boffetta $P$, et al (2013) Hierarchical modeling identifies novel lung cancer susceptibility variants in inflammation pathways among 10,140 cases and 11,012 controls. Human genetics 132:579-89

5. Ji X, Bossé $Y$, Landi MT, et al (2018) Identification of susceptibility pathways for the role of chromosome 15q25.1 in modifying lung cancer risk. Nature Communications 9:3221

6. Truong T, Sauter W, McKay JD, et al (2010) International Lung Cancer Consortium: coordinated association study of 10 potential lung cancer susceptibility variants. Carcinogenesis 31:625-633

7. Wang Y, Wei Y, Gaborieau V, et al (2015) Deciphering associations for lung cancer risk through imputation and analysis of 12316 cases and 16831 controls. European Journal of Human Genetics 23:1723-1728

8. Feng $\mathrm{Y}$, Wang $\mathrm{Y}$, Liu $\mathrm{H}$, et al (2018) Novel genetic variants in the P38MAPK pathway gene ZAK and susceptibility to lung cancer. Mol Carcinog 57:216-224

9. Landi MT, Chatterjee N, Yu K, et al (2009) A genome-wide association study of lung cancer identifies a region of chromosome $5 \mathrm{p} 15$ associated with risk for adenocarcinoma. American journal of human genetics 85:67991

10. Zhan T, Rindtorff $\mathrm{N}$, Boutros $\mathrm{M}$ (2016) Wnt signaling in cancer. Oncogene. https://doi.org/10.1038/onc.2016.304

11. Buniello A, MacArthur JAL, Cerezo M, et al (2019) The NHGRI-EBI GWAS Catalog of published genome-wide association studies, targeted arrays and summary statistics 2019. Nucleic Acids Res 47:D1005-D1012

12. (2020) GWAS catalog. In: The NHGRI-EBI Catalog of human genome-wide association studies. https://www.ebi.ac.uk/gwas/. Accessed 22 Dec 2020

13. Kerdidani D, Chouvardas P, Arjo AR, et al (2019) Wnt1 silences chemokine genes in dendritic cells and induces adaptive immune resistance in lung adenocarcinoma. Nat Commun. https://doi.org/10.1038/s41467-01909370-z

14. Fang L, Cai J, Chen B, et al (2015) Aberrantly expressed miR-582-3p maintains lung cancer stem cell-like traits by activating Wnt/beta-catenin signalling. Nat Commun 6:8640

15. Amos $\mathrm{Cl}, \mathrm{Wu} X$, Broderick $\mathrm{P}$, et al (2008) Genome-wide association scan of tag SNPs identifies a susceptibility locus for lung cancer at 15q25.1. Nat Genet 40:616-622

16. Yuan Y, Lu C, Xue L, Ge D (2014) Association between TERT rs2736100 polymorphism and lung cancer susceptibility: evidence from 22 case-control studies. Tumor Biol 35:4435-4442

17. Akiyama T (2000) Wnt/beta-catenin signaling. Cytokine Growth Factor Rev 11:273-82 
18. Schneider AJ, Branam AM, Peterson RE (2014) Intersection of AHR and Wnt Signaling in Development, Health, and Disease. International Journal of Molecular Sciences 15:17852-17885

19. Chang JT, Chang H, Chen PH, Lin SL, Lin P (2007) Requirement of aryl hydrocarbon receptor overexpression for CYP1B1 up-regulation and cell growth in human lung adenocarcinomas. Clin Cancer Res 13:38-45

20. Lin P, Chang H, Tsai WT, Wu MH, Liao YS, Chen JT, Su JM (2003) Overexpression of aryl hydrocarbon receptor in human lung carcinomas. Toxicol Pathol 31:22-30

21. Wang CK, Chang H, Chen PH, Chang JT, Kuo YC, Ko JL, Lin P (2009) Aryl hydrocarbon receptor activation and overexpression upregulated fibroblast growth factor-9 in human lung adenocarcinomas. Int J Cancer 125:80715

22. Bahl C, Singh N, Behera D, Sharma S (2017) High-order gene interactions between the genetic polymorphisms in Wnt and AhR pathway in modulating lung cancer susceptibility. Personalized Medicine. https://doi.org/10.2217/pme-2017-0018

23. Bahl C, Singh N, Behera D, Sharma S (2017) Association of polymorphisms in Dickopff (DKK) gene towards modulating risk for lung cancer in north Indians. Future Oncol 13:213-232

24. Yilmaz M, Donmez G, Kacan T, Sari I, Akgül Babacan N, Sari M, Kilickap S (2015) Significant Association Between Polymorphisms of Wnt Antagonist Genes and Lung Cancer: Journal of Investigative Medicine 1

25. Amos Cl, Dennis J, Wang Z, et al (2017) The OncoArray Consortium: A Network for Understanding the Genetic Architecture of Common Cancers. Cancer Epidemiol Biomarkers Prev 26:126-135

26. Machiela MJ, Chanock SJ (2015) LDlink: a web-based application for exploring population-specific haplotype structure and linking correlated alleles of possible functional variants. Bioinformatics 31:3555-3557

27. Chang CC, Chow CC, Tellier LC, Vattikuti S, Purcell SM, Lee JJ (2015) Second-generation PLINK: rising to the challenge of larger and richer datasets. GigaScience 4:1-16

28. Purcell S, Neale B, Todd-Brown K, et al (2007) PLINK: a tool set for whole-genome association and populationbased linkage analyses. American journal of human genetics 81:559-75

29. Stone M (1977) An Asymptotic Equivalence of Choice of Model by Cross-Validation and Akaike's Criterion. Journal of the Royal Statistical Society Series B (Methodological) 39:44-47

30. Fang Y (2009) Asymptotic Equivalence between Cross-Validations and Akaike Information Criteria in MixedEffects Models. JDS 15-21

31. DeLong ER, DeLong DM, Clarke-Pearson DL (1988) Comparing the areas under two or more correlated receiver operating characteristic curves: a nonparametric approach. Biometrics 44:837-845

32. Andri S (2021) DescTools: Tools for Descriptive Statistics.

33. Therneau T, Atkinson B, Ripley B (2019) rpart: Recursive partitioning for classification, regression and survival trees.

34. Uhlén M, Fagerberg L, Hallström BM, et al (2015) Tissue-based map of the human proteome. Science. https://doi.org/10.1126/science.1260419

35. The Human Protein Atlas. https://www.proteinatlas.org/. Accessed 5 Feb 2021 
37. Du Y, Guo M, Whitsett JA, Xu Y (2015) "LungGENS": a web-based tool for mapping single-cell gene expression in the developing lung. Thorax 70:1092-1094

38. Xue P, Fu J, Zhou Y (2018) The Aryl Hydrocarbon Receptor and Tumor Immunity. Front Immunol. https://doi.org/10.3389/fimmu.2018.00286

39. Xu H, Wu J, Chen B, et al (2014) Serum Dickkopf-1 (DKK1) is significantly lower in patients with lung cancer but is rapidly normalized after treatment. Am J Transl Res 6:850-856

40. Shen T, Chen Z, Qiao J, Sun X, Xiao Q (2019) Neutralizing monoclonal antibody against Dickkopf2 impairs lung cancer progression via activating NK cells. Cell Death Discovery 5:1-12

41. Reich D, Thangaraj K, Patterson N, Price AL, Singh L (2009) Reconstructing Indian Population History. Nature 461:489-494

42. Lee PN, Forey BA, Coombs KJ (2012) Systematic review with meta-analysis of the epidemiological evidence in the 1900 s relating smoking to lung cancer. BMC cancer 12:385

43. Katki HA, Kovalchik SA, Petito LC, Cheung LC, Jacobs J, Jemal A, Berg CD, Chaturvedi AK (2018) Implications of Nine Risk Prediction Models for Selecting Ever-Smokers for Computed Tomography Lung Cancer Screening. Annals of internal medicine. https://doi.org/10.7326/M17-2701

44. Jia G, Lu Y, Wen W, Long J, Liu Y, Tao R, Li B, Denny JC, Shu X-O, Zheng W (2020) Evaluating the Utility of Polygenic Risk Scores in Identifying High-Risk Individuals for Eight Common Cancers. JNCl Cancer Spectr. https://doi.org/10.1093/jncics/pkaa021

45. Jia G, Wen W, Massion PP, Shu X-O, Zheng W (2021) Incorporating Both Genetic and Tobacco Smoking Data to Identify High-Risk Smokers for Lung Cancer Screening. Carcinogenesis. https://doi.org/10.1093/carcin/bgab018

46. Hung RJ, Warkentin MT, Brhane Y, et al (2021) Assessing Lung Cancer Absolute Risk Trajectory Based on a Polygenic Risk Model. Cancer Res 81:1607-1615

47. Li Y, Xiao X, Bossé Y, et al (2019) Genetic interaction analysis among oncogenesis-related genes revealed novel genes and networks in lung cancer development. Oncotarget 10:1760-1774

48. Sun S, Schiller JH, Gazdar AF (2007) Lung cancer in never smokers--a different disease. Nature reviews Cancer 7:778-90

49. Saccone NL, Culverhouse RC, Schwantes-An T-H, et al (2010) Multiple Independent Loci at Chromosome 15q25.1 Affect Smoking Quantity: a Meta-Analysis and Comparison with Lung Cancer and COPD. PLOS Genetics 6:e1001053

50. Liu CY, Stucker I, Chen C, Goodman G, McHugh MK, D’Amelio AM Jr, Etzel CJ, Li S, Lin X, Christiani DC (2015) Genome-wide Gene-Asbestos Exposure Interaction Association Study Identifies a Common Susceptibility Variant on 22q13.31 Associated with Lung Cancer Risk. Cancer Epidemiol Biomarkers Prev 24:1564-73

51. Wei S, Wang L-E, McHugh MK, Han Y, Xiong M, Amos Cl, Spitz MR, Wei QW (2012) Genome-wide gene-environment interaction analysis for asbestos exposure in lung cancer susceptibility. Carcinogenesis 33:1531-1537 
583

584

585

586

587

588

589

590

591

592
52. Rosenberger A, Hung RJ, Christiani DC, et al (2018) Genetic modifiers of radon-induced lung cancer risk: a genome-wide interaction study in former uranium miners. Int Arch Occup Environ Health. https://doi.org/10.1007/s00420-018-1334-3

53. Lorenzo-González M, Ruano-Ravina A, Torres-Durán M, et al (2019) Residential radon, genetic polymorphisms in DNA damage and repair-related. Lung Cancer 135:10-15

54. Hung RJ, Spitz MR, Houlston RS, et al (2019) Lung Cancer Risk in Never-Smokers of European Descent is Associated With Genetic Variation in the 5p15.33 TERT-CLPTM1LI Region. J Thorac Oncol 14:1360-1369

55. Du Y, Kitzmiller JA, Sridharan A, et al (2017) Lung Gene Expression Analysis (LGEA): an integrative web portal for comprehensive gene expression data analysis in lung development. Thorax 72:481-484 


\section{Supplementary Files}

This is a list of supplementary files associated with this preprint. Click to download.

- DKKSupplementV4.1ohneVerweise.pdf 